\title{
Influence of anterior mitral valve length and septal wall thickness on the prevalence of left ventricular outflow tract obstruction in hypertrophic cardiomyopathy
}

\author{
Kareem Morant ${ }^{1 *}$, John Stirrat ${ }^{3}$, David Scholl ${ }^{3}$, Maria Drangova ${ }^{3}$, James A White ${ }^{2,3}$ \\ From 15th Annual SCMR Scientific Sessions \\ Orlando, FL, USA. 2-5 February 2012
}

\section{Summary}

In this study, we aimed to identify the respective and combined influences of AMVL length and septal thickening on the development of LVOT obstruction in a population of patients with HCM.

\section{Background}

Interest has emerged in the evaluation of mitral valve morphology in patients with Hypertrophic Cardiomyopathy $(\mathrm{HCM})$ and its contribution to left ventricular outflow tract obstruction (LVOTO). Specifically, elongation of the anterior mitral valve leaflet (AMVL) appears to be part of phenotypic expression in $\mathrm{HCM}$ and has been associated with severity of LVOT obstruction. However, the influence of septal thickening and its physiologic interaction with AMVL elongation is not well understood.

\section{Methods}

Consecutive HCM patients referred for Cardiac MRI evaluation between March 2008 and May 2011 were identified from a prospective clinical registry. All patients underwent a standardized imaging protocol inclusive of cine imaging and phase-contrast flow imaging. Cine imaging was performed in sequential short axis planes and in the 2, 3 and 4-chamber views. Phase contrast flow of the LVOT was performed to identify the peak systolic velocity. All images were analyzed using commercially available software (CMR42, Circle Cardiovascular Inc., Calgary). Cine images were blindly

'Deparment of Medicine, University of Western Ontario, London, ON, Canada Full list of author information is available at the end of the article analyzed for AMVL length, septal wall thickness (SWT), LVOT diameter, LV chamber volumes and stroke volume. A novel calculated measure was also evaluated, the "Septal Anterior Mitral Valve Length Product" (SALP), determined by the multiplying of SWT and AMVL length. All patients with an LVOT pressure gradient $\geq 30 \mathrm{mmHg}$ were defined as having LVOTO.

\section{Results}

A total of 75 patients were studied with a mean age of $54 \pm 14$ years. The mean maximal wall thickness was $19.5 \pm 5.5 \mathrm{~mm}$. Among all baseline clinical and MRI characteristics SWT and SALP were the only significant predictors of resting LVOTO, occurring in 15 (19.2\%) patients (Figure 1). By Receiver Operator Characteristics (ROC) analysis SALP demonstrated superior predictive accuracy for the occurrence of LVOTO (AUC=0.79) (Figure 2) compared with SWT alone (AUC=0.71). The sensitivity and specificity of SALP for prediction of obstruction was $73 \%$ and $87 \%$, respectively.

\section{Conclusions}

This study identifies a combined influence of AMVL length and SWT on the development of LVOTO in HCM. The product of these two measures, a marker we term SALP, may be a useful tool to identify early clinical phenotypes of HCM and those at higher likelihood of dynamic LVOTO.

\section{Funding}

J.A.W is a clinician scientist with the Heart and Stroke Foundation of Ontario, Canada. This research was 


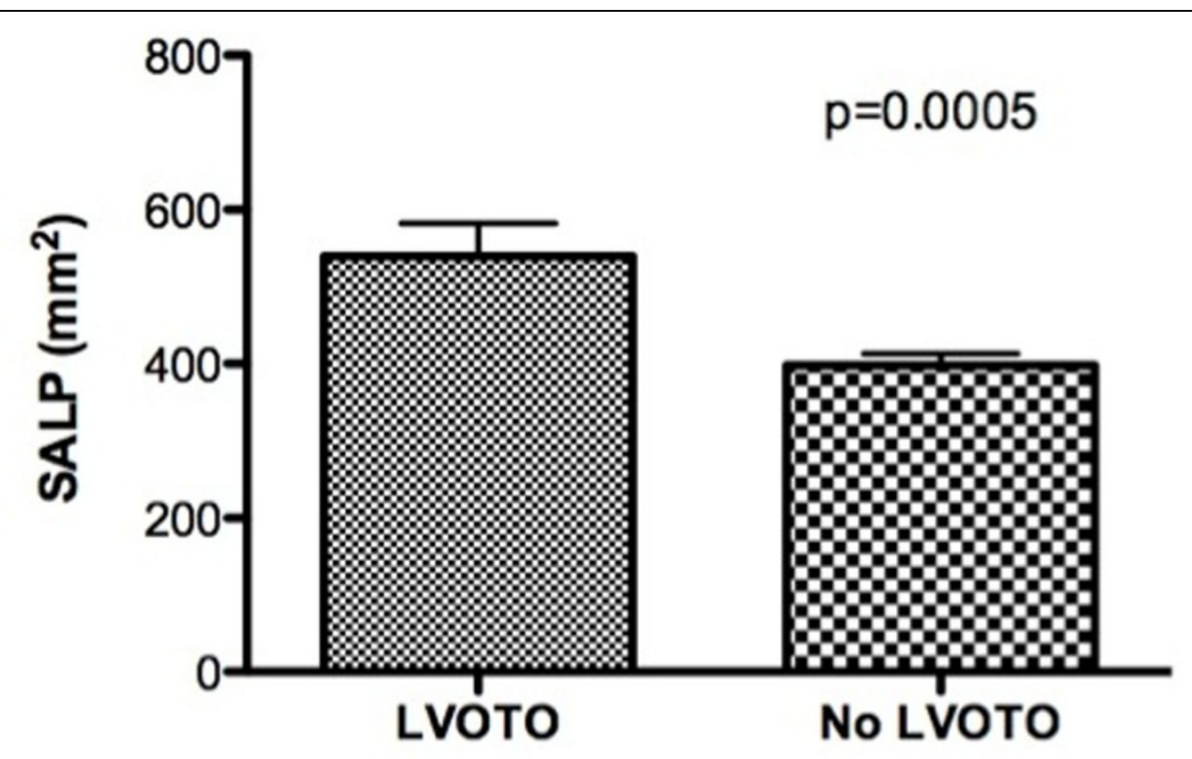

Figure 1

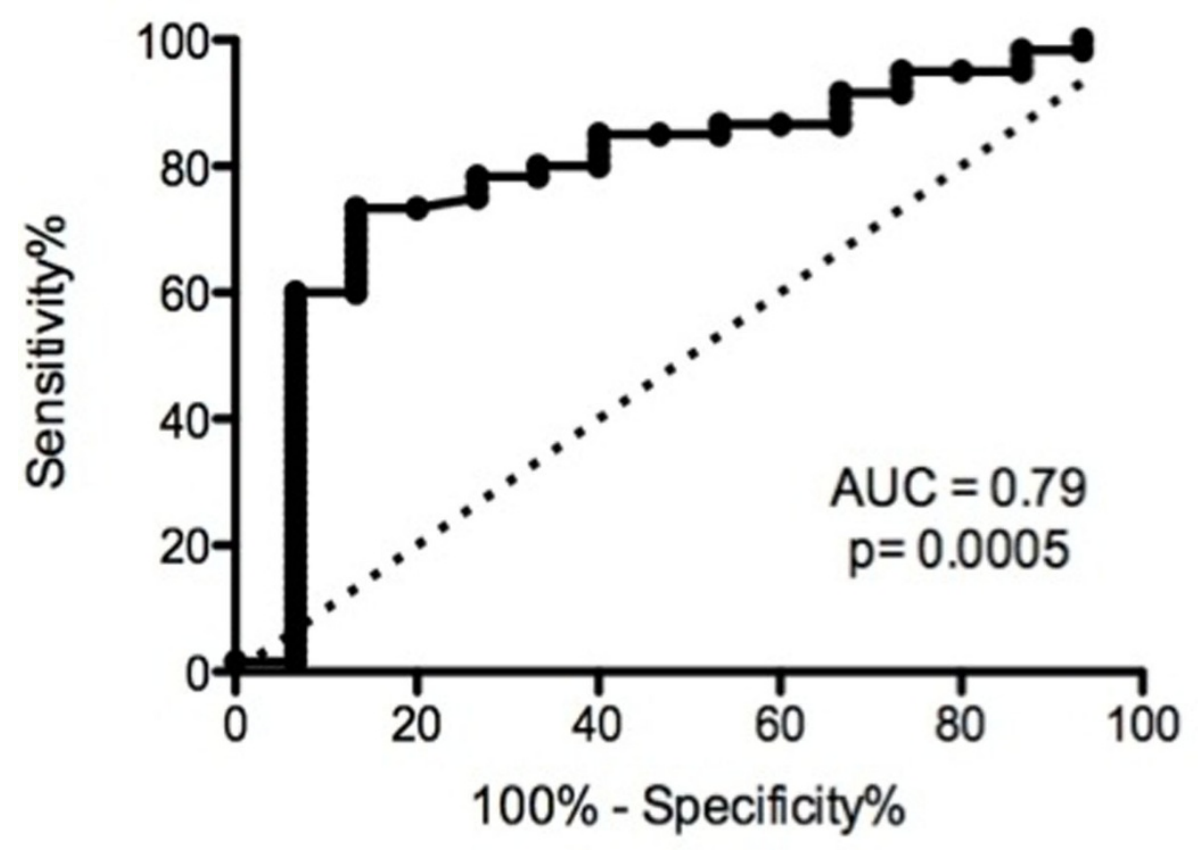

Figure 2

supported by the Heart and Stroke Foundation grant \# NA6488 (PI: J.A.W.) and by the Canada

Foundation of Innovation (CFI) Leaders Opportunity Fund.

\section{Author details}

'Deparment of Medicine, University of Western Ontario, London, ON, Canada. ${ }^{2}$ Division of Cardiology, Department of Medicine, London Health Sciences Centre, London, ON, Canada. ${ }^{3}$ Imaging Research Labratory-Robarts Research Institute, University of Western Ontario, London, ON, Canada.

Published: 1 February 2012 
doi:10.1186/1532-429X-14-S1-P169

Cite this article as: Morant et al:: Influence of anterior mitral valve length and septal wall thickness on the prevalence of left ventricular outflow tract obstruction in hypertrophic cardiomyopathy. Journal of Cardiovascular Magnetic Resonance 2012 14(Suppl 1):P169.

Submit your next manuscript to BioMed Central and take full advantage of:

- Convenient online submission

- Thorough peer review

- No space constraints or color figure charges

- Immediate publication on acceptance

- Inclusion in PubMed, CAS, Scopus and Google Scholar

- Research which is freely available for redistribution

Submit your manuscript at www.biomedcentral.com/submit 